\title{
Twin Occlusion in Maxillary Overdenture with Access Posts and O-ring Attachments in the Rehabilitation of Hemimandibulectomy Patient
}

\author{
${ }^{1}$ KV Arun Kumar, ${ }^{2}$ Virender S Legha, ${ }^{3}$ Dinesh K Saini, ${ }^{4}$ Vinay N Wadhwa, ${ }^{5}$ Abir Sarkar, ${ }^{6}$ Pramod K Chahar
}

\begin{abstract}
Discontinuity of mandible limits the balance of the lower face and leads to decreased mandibular function by deviation of residual segment toward surgical site. Delay in the initiation of mandibular guidance therapy may be due to varied reasons like postsurgical morbidities, tight wound closure, radiation therapy, flap necrosis, and others, which may result in an inability to achieve normal maxillomandibular relationships. When such deviated mandible is opposed to maxillary complete denture, then the rehabilitation would be of greater challenge. This clinical report presents rehabilitation of hemimandibulectomy defect with twin occlusion in maxillary overdenture with access posts and O-ring attachments.
\end{abstract}

Keywords: Access post, Hemimandibulectomy, Twin occlusion.

How to cite this article: Kumar KVA, Legha VS, Saini DK. Wadhwa VN, Sarkar A, Chahar PK. Twin Occlusion in Maxillary Overdenture with Access Posts and O-ring Attachments in the Rehabilitation of Hemimandibulectomy Patient. Int J Experiment Dent Sci 2017;6(2):105-107.

Source of support: Nil

Conflict of interest: None

\section{INTRODUCTION}

Mastication and other oral functions are achieved by coordinated movement of mandible against maxillae. Temporomandibular joints and muscles involved in mastication play a pivotal role in effective and harmonized jaw movements. Hemimandibulectomy is a surgical procedure where one half of the mandible is resected, usually as a result of management of oral malignancy involving mandible. Unilateral discontinuity of mandible due to surgery or trauma results in the deviation of mandible toward the defect side. The cause for deviation of mandible is multifactorial, which includes extent and site of the resection, tissue contracture, and innervation involved. ${ }^{1}$

\footnotetext{
${ }^{1,2}$ Reader, ${ }^{3}$ Associate Professor, ${ }^{4-6}$ Resident

${ }^{1-6}$ Department of Prosthodontics, Armed Forces Medical College, Pune, Maharashtra, India

Corresponding Author: KV Arun Kumar, Reader, Department of Prosthodontics, Armed Forces Medical College, Pune Maharashtra, India, Phone: +912026332931, e-mail: prarun2009 @gmail.com
}

Management of hemimandibulectomy patients is often challenging and involves multispecialty approach. Proper planning and consultation between operating surgeon and maxillofacial prosthodontist is vital in rehabilitating the patient. To achieve maximum function and esthetics, early prosthodontic consultation is essential. ${ }^{2}$ Presurgical records can help the prosthodontist in fabrication of mandible guiding appliance. Mandibular deviation after hemimandibulectomy can be minimized using numerous techniques. ${ }^{1}$ In spite of all these efforts, some amount of deviation still occurs and a dual occlusion is given. This study presents the management of a patient with hemimandibular defect patient with slight mandibular deviation with twin occlusal scheme.

\section{CASE REPORT}

A 65-year-old male patient was referred to the prosthodontic outpatient department for the replacement of missing teeth. His past medical and dental history revealed that hemimandibulectomy was done 2 years back for surgical management of squamous cell carcinoma of right buccal mucosa invading into the mandible (Fig. 1). Mandible guide flange appliance was provided for initial 6 months postsurgery. This was followed by radiotherapy for 3 months. Some teeth with poor prognosis were extracted a year back. Since last 1 year, he was partially edentulous in maxilla and mandible. His extraoral examination revealed asymmetrical face, reduced lower facial height, and competent lips with contracture of right lower lip. On palpation, mandible was palpable until midline and absent on right side. Total of eight teeth were present $(11,21,22$, $23,32,33,34,35)$. Deviation of mandible toward the defect side was evident. His speech was distorted. Collaborating

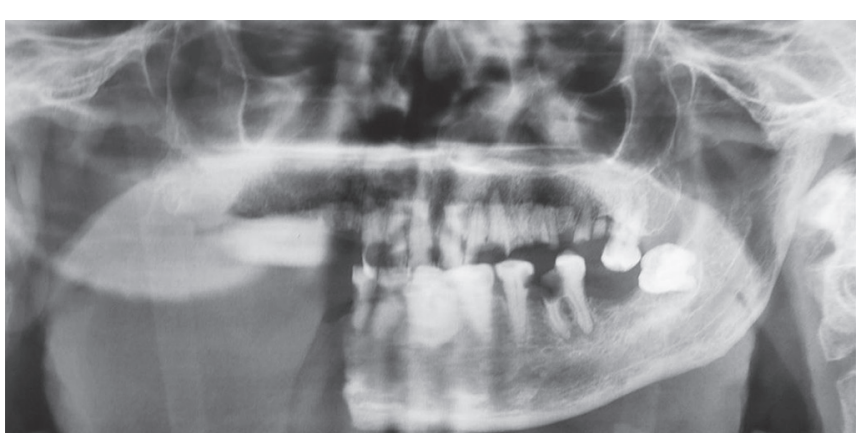

Fig. 1: Orthopantomograph showing hemimandibulectomy defect 
the patient's history, clinical findings, and investigations, it was diagnosed as partially edentulous maxillary arch (Kennedy's class I) and mandibular arch (class II Cantor and Curtis hemimandibulectomy defect-right). The treatment objective was to establish oral function, appearance, and psychological reassurance to the patient. After consultations, treatment plan was made to fabricate access postretained maxillary overdenture with twin occlusion on the unresected side and cast partial denture in the mandibular arch to replace 41,31,36, 37. Oral prophylaxis, restoration of carious teeth, and endodontic treatment of 11, 21, 22, 23 were done. After completion of phase I and II therapy, tooth preparation of endodontic treated teeth was done to receive metal copings on 21,23 and metal copings with access holes on 11,22. Copings were cemented using resin-reinforced glass-ionomer cement (Fig. 2A).

Primary impressions were made using putty-light body elastomeric impression material. Special tray for maxillary arch was fabricated with the window in the abutment region and tray handle in palate region. Border molding was done and secondary impressions were recorded in elastomeric impression material. Medium body addition silicone was injected over the abutments in the window created in the special tray and type II dental stone was placed with a brush over the medium body silicone on the outer surface and impression was retrieved and cast fabricated. After mouth preparation, mandibular final impression was recorded using dual impression technique. Cast metal denture base for the maxillary arch (Fig. 2B) and cast partial framework for mandibular arch were fabricated. Jaw relations were recorded using Jet bite (Coltene Whaledent) silicone interocclusal records. Occlusal rim of the maxillary arch on unresected side was broadened to record the deviated path of mandible.

Teeth arrangement was done using semi-anatomic teeth. For maxillary arch on the unresected side, teeth arrangement was done in two rows. Outer row of teeth was arranged to achieve esthetics and support. Inner row of nonanatomic teeth was arranged on the palatal side of initial set teeth according to deviated path of the mandible to achieve occlusion. The mandibular teeth on the unresected side were articulated with the inner row of the maxillary denture teeth. The trial dentures were checked in patient's mouth and occlusion, phonetics, and esthetics ascertained. The dentures were processed in heat cure polymethylmethacrylate resin using compression molding technique (Fig. 3A). Processed dentures were checked in the patient's mouth and occlusal interferences were corrected.

Access posts were cemented on the teeth 11 and 22 after root canal space preparation (Fig. 3B). O-rings along with housings were placed on the studs of the access posts. Using window technique, housings were picked up with autopolymerizing resin into the denture (Fig. 3C). Prostheses were inserted (Fig. 4) after evaluation of retention, stability, and occlusion. Postprostheses insertion instructions were
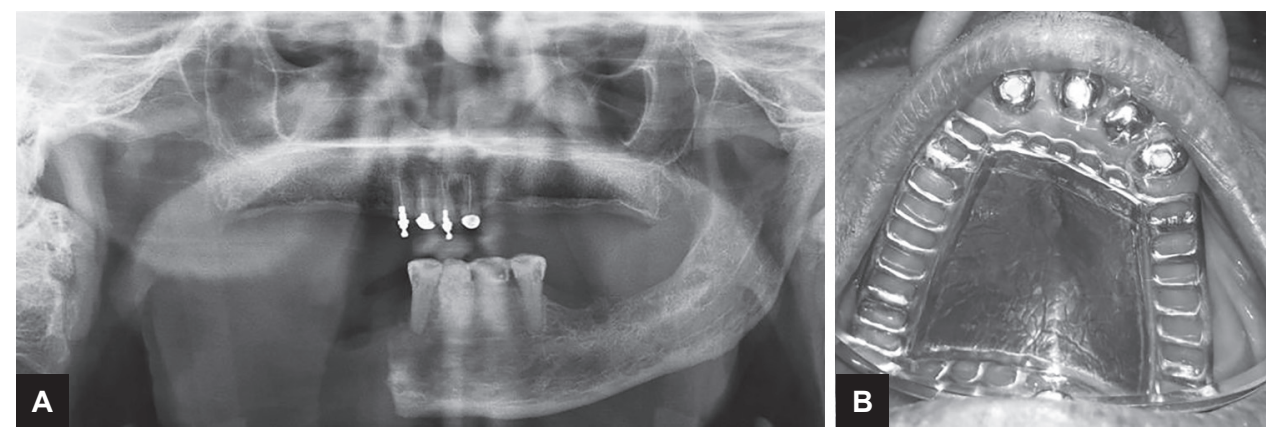

Figs 2A and B: (A) Posttreatment orthopantomogram; (B) cemented metal copings and cast metal denture base try-in
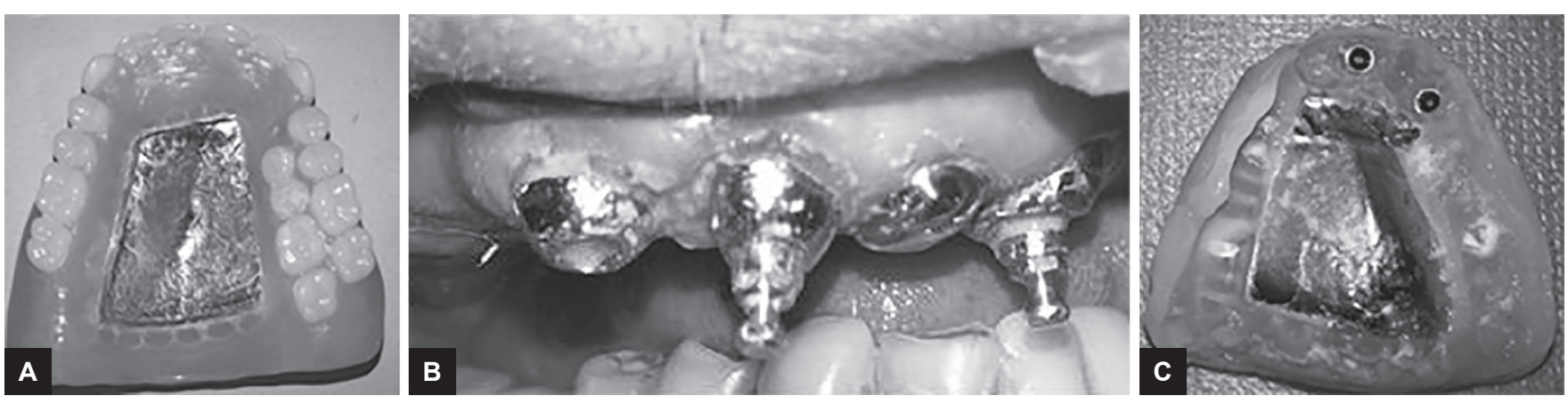

Figs 3A to C: (A) Finished maxillary overdenture with twin occlusion; (B) access post with O-ring and housing; and (C) impression surface of maxillary overdenture with picked up O-ring 

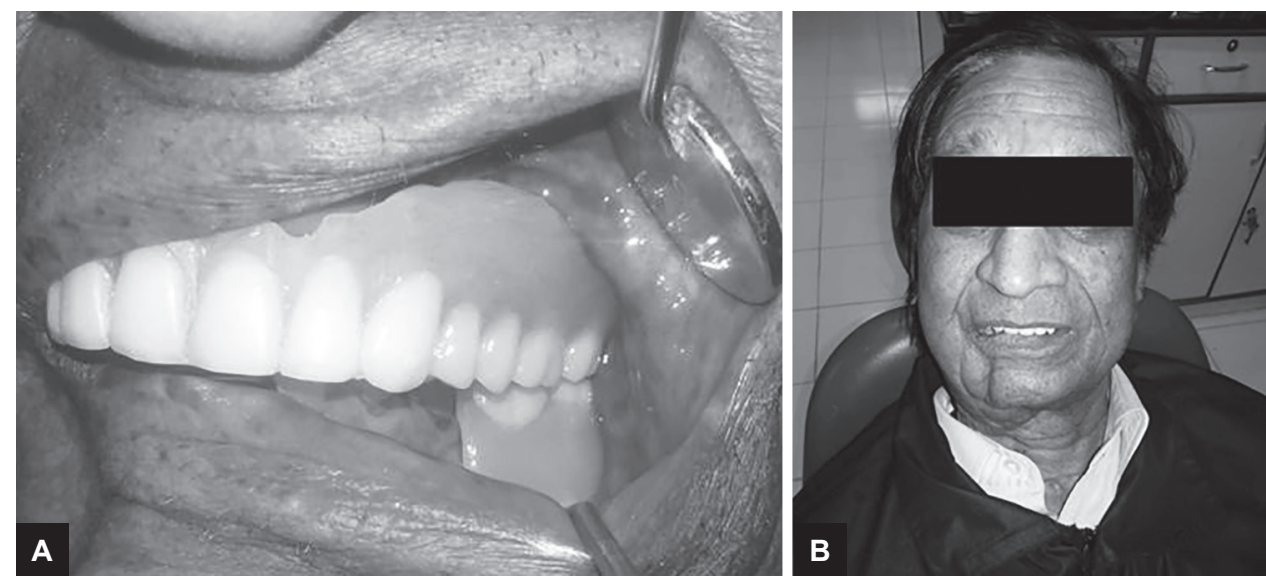

Figs 4A and B: (A) Occlusion on unaffected side; (B) posttreatment

explained verbally to the patient and a written set of instructions was also provided. Patient was evaluated after 48 hours, 7 days, 1 month, and thereafter every 3 months. Postinsertion problems or discomfort were addressed. Postoverdenture therapy, patient's mastication, esthetics, and phonetics were satisfactory.

\section{DISCUSSION}

Unreconstructed mandibular defects generally pose rehabilitative challenges ${ }^{3-5}$ due to reduced mouth opening, loss of vestibule following resective surgery, deviation of mandible, uncontrolled tongue movements or tongue attached as flap, as well as sequel of surgical scar contracture and radiotherapy side effects. If surgical reconstruction is not possible, then the prosthodontic management would be the treatment of choice. ${ }^{6}$ Deviation of mandible toward the defect side can be prevented by initial surgical reconstruction, using guide flange or palatal ramp appliance, initial intermaxillary fixation postsurgery, and guided closure excercises. ${ }^{7}$ In spite of all these preventive measures, the mandible may get slightly deviated during functional movements. Hence, in the final prosthodontic rehabilitation, guide flange can be incorporated in the metal framework of the prosthesis ${ }^{8}$ or twin occlusion can be provided on maxillary denture to accommodate the deviation. ${ }^{9}$ Arranging the teeth in the neutral zone would also increase the stability of the prosthesis. ${ }^{10}$ In the present case report, the second row of nonanatomic teeth was placed palatal to the regular teeth arrangement on the unresected side. This allowed good occlusal intercuspation during mandibular closure. Unilateral unbalanced forces resultant to mandibular deviation tend to unseat the maxillary complete denture. Hence, in the present case, access posts with O-ring attachments facilitated in providing additional retention and stability to the maxillary denture during function. However, increased thickness in the anterior region of maxillary denture, requirement to change of O-rings to enhance retention, and regular fluoride application around the abutment teeth to prevent dental caries are few drawbacks of using access posts and O-ring attachments used to retain dentures.

\section{CONCLUSION}

This case report presents rehabilitation of hemimandibulectomy defect with slight deviation using twin occlusion. The retention of the maxillary overdenture was reinforced with access posts and O-ring attachments. Regular checkup and motivation help the patient to adapt to new dentures. These rehabilitative efforts would facilitate in restoring form, function, esthetics, and psychosocial well-being of the patient.

\section{REFERENCES}

1. Schneider RL, Taylor TD. Mandibular resection guidance prosthesis: a literature review. J Prosthet Dent 1986 Jan;55(1):84-86.

2. Edgerton MT Jr, Pyott JE. Surgery and prosthesis in jaw reconstruction. J Prosthet Dent 1954 Mar;4(2):257-262.

3. Swoope CC. Prosthetic management of resected edentulous mandibles. J Prosthet Dent 1969 Feb;21(2):197-202.

4. Mankar S, Pakhan A, Thombore R, Godbole S. Twin occlusion: a prosthetic management of hemimandibulectomy patient $-\mathrm{a}$ case report. NJMDR 2012;1(1):19-23.

5. Taylor, TD. Diagnostic considerations for prosthodontics rehabilitation of the mandibulectomy patient. In: Taylor TD, editor. Clinical maxillofacial prosthetics. Chicago (IL): Quintessence; 2000. p. 155-170.

6. Emory L. Partial denture prosthesis for war injuries. J Am Dent Assoc 1947 Nov;35(9):634-644.

7. Beumer, J III.; Curtis, TA.; Marunick, MT. Maxillofacial rehabilitation: prosthodontic and Surgical consideration. 3rd ed. St. Louis (MO): Ishiyaku Euro America; 1996.

8. Chalian, VA.; Drane, JB.; Standish, SM. Maxillofacial prosthesis: multidisciplinary practice. Baltimore (MD): The William \& Wilkins Co; 1972.

9. Rosenthal LE. The edentulous patient with jaw defects. Dent Clin North Am 1994;8:773-779.

10. Atreja G. Prosthetic rehabilitation of partially resected edentulous mandible. IJCCI 2011;3:12-16 